Max-Planck-Institut für demografische Forschung

Max Planck Institute for Demographic Research

Konrad-Zuse-Strasse 1 - D-18057 Rostock = Germany = Tel +49 (0) 3812081 - 0 - Fax +49 (0) 3812081 - 202 - www.demogr.mpg.de

\title{
hmddata - A Stata Module to Access and Work with Data from the Human Mortality Database
}

Daniel C. Schneider I schneider@demogr.mpg.de

For additional material see www.demogr.mpg.de/tr

This technical report has been approved for release by: Mikko Myrskylä (sekmyrskyla@demogr.mpg.de), Head of the Laboratory of Population Health and Head of the Laboratory of Fertility and Well-Being \& Vladimir Shkolnikov (shkolnikov@demogr.mpg.de), Head of the Laboratory of Demographic Data.

(c) Copyright is held by the authors.

Technical reports of the Max Planck Institute for Demographic Research receive only limited review. Views or opinions expressed in technical reports are attributable to the authors and do not necessarily reflect those of the Institute. 


\title{
hmddata - A Stata Module to Access and Work with Data from the Human Mortality Database
}

\author{
Daniel C. Schneider \\ Max Planck Institute for Demographic Research, Rostock, Germany
}

June 6, 2017 (updated July 11, 2018)

\begin{abstract}
Assembling a data set for a particular research question from the Human Mortality Database (HMD) can be cumbersome. Different tools exist for facilitating this task in $\mathrm{R}$. This report describes a Stata add-on module, called "hmddata", that facilitates importing and working with HMD data in Stata. It can import almost all of HMD statistics in an easy and flexible manner. It can also be used to accomplish common data tasks, and allows for the easy creation of tables and graphs.
\end{abstract}

\section{Motivation}

The Human Mortality Database offers a rich set of demographic statistics, most notably mortality statistics, and is a widely used data source in demographic and health research, and in the social sciences in general. While its usage is free of charge, the technical data acquisition can be cumbersome, since the full data offering is scattered across $7000+$ text files. Compiling a data set geared towards a particular research question can be a tedious task. It would therefore be desirable to have tools that facilitate the data import.

Such tools currently exist only for R. Available R packages are:

- HMDHFDplus (Riffe 2015): accesses files for any statistic offered by the HMD.

- Demography (Hyndman 2014): function hmd. mx () accesses files for mortality rates, population counts, and life expectancy at birth (1x1 year-by-age grid only).

The above packages access single (country-specific) text files over the web.

In addition, two MPIDR technical reports provide R scripts that access HMD data:

- Scripts by Shkolnikov/Jdanov (2016) import life table data and death rates for all countries. 
- Scripts by Minton (2015) import death counts and population counts (1x1 year-by-age grid only) for all countries.

The R scripts are based on text files contained in all-country zip files from the HMD website.

This technical report introduces a HMD data tool, called "hmddata", that complements and expands on the existing tools in several respects:

- It is written in Stata, which is, like $R$, a program in widespread use within the social sciences.

- It can access HMD statistics in an easy and efficient manner.

- It provides tools for working with the data that go beyond mere import functionality.

Details on the features and capabilities of hmddata are provided in the next section.

hmddata comes with extensive Stata help entries. Since there is no need to duplicate this information here, this report will only give a terse description of the features of hmddata and provide installation instructions. The Stata help entries, which contain much more detail than this report, can be accessed after installation by issuing in Stata

\section{- help hmddata}

or even without installation by issuing in Stata

- net from https://user.demogr.mpg.de/schneider/stata

and then following the point-and-click interface to the remote hmddata help entries. This report concludes with an examples section that demonstrates the usefulness of hmddata.

\section{Features and Capabilities of hmddata}

The development goals of hmddata were:

1. provide convenient access to all HMD data

2. provide data management tools for common tasks

3. allow quick and easy generation of tables and graphs whose quality is sufficient for working stage paper drafts and communication among coauthors

The first goal led to the following features of hmddata:

- It can process almost all HMD data. The only exception are Lexis input DB files. ${ }^{1}$

- It can process any zip file that can be downloaded from the HMD website. ${ }^{2}$

- It covers all statistics, all countries, all age-by-year grids.

- It can convert/access data selectively: individual or multiple or all countries, individual or multiple or all statistics, individual or multiple or all age-by-year grids.

- It stores data efficiently. ${ }^{3}$

\footnotetext{
${ }^{1}$ For the sake of simplicity, the following text will refer to the data scope of hmddata as being "all HMD data". The limitation of Lexis input DB files not being included is implicitly understood.

${ }^{2}$ The zip files are located at http://www.mortality.org/cgi-bin/hmd/hmd download.php.

${ }^{3}$ For example, the contents of the comprehensive by-statistics zip file takes up around 600MB (as of April 11, 2017). The Stata files that contain these data are only $220 \mathrm{MB}$ large.
} 
Goals number two and three are achieved by:

- separate subcommands for common HMD data tasks: generation of interval variables, filtering populations, graphing

- the usage of data labels, variable labels, value labels, and other descriptive information

Moreover, as is the case with all Stata add-on modules, installation, updating, and deinstallation is very easy. Finally, the module's workings and options are described in great detail in the associated Stata help files.

\section{Basic Ideas}

hmddata processes zip files that can be downloaded from the HMD website once a user has registered. ${ }^{4}$ hmddata itself does not connect to HMD data over the web. It accesses files that have been downloaded manually. After downloading one or more zip files and the extraction of their contents, the first step is to convert the source text files into Stata files. hmddata convert accomplishes this. It saves the Stata HMD files to a directory that has been specified using hmddata settings. hmddata use then can properly access the generated Stata files and load HMD data sets into memory. Subcommands intervals, popfilter, and graph can be used for frequent data management tasks. hmddata files and hmddata info are small helper functions.

\section{Installation and Code Updates}

Stata 14, which has been released in April 2015, or a later Stata version is required for installation. ${ }^{5}$ hmddata can be installed on all systems that Stata runs on (Windows, Mac, Linux). Note that all Stata files (and therefore all files created by hmddata) are fully portable across supported platforms. Testing, however, has only been done under Windows.

The hmddata module can be installed from within Stata by

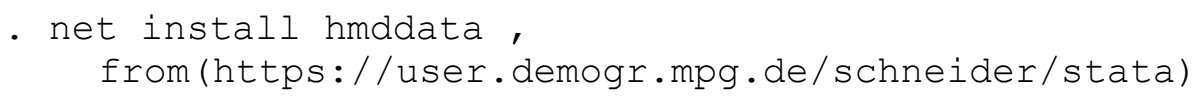

Make sure to use the https (and not: http) protocol as in the command above. Updates to the program can be installed by

- adoupdate hmddata

Deinstallation is done by

- ado uninstall hmddata

If direct installation over the web is not possible, one can also download https://user.demogr.mpg.de/schneider/stata/hmddata/misc/hmddata.zip, unzip the file contents,

\footnotetext{
${ }^{4}$ The zip files are located at http://www.mortality.org/cgi-bin/hmd/hmd download.php.

${ }^{5}$ hmddata in principle also runs under Stata 13, but unfortunately this Stata version has been reported to sometimes generate errors during installation. The exact source of these errors is not known, but may be related to the https protocol used for the web install. Moreover, in Stata 13, using reshape with option j (age) may result in an error, due to a Stata bug which has been fixed with the 06may2014 update. All of these issues are no longer present in Stata 14 and higher.
} 
and follow the instructions in the text file readme_zipinstall.txt. ${ }^{6}$ The downside of this installation method is that it does not provide a convenient updating mechanism. Code updates are best performed by deinstallation of the package, download of the updated zip file, and local reinstallation.

\section{Usefulness for Other Software Environments}

hmddata may also be useful if you work with a different software than Stata. If you are willing to go through the text-to-Stata-files conversion process in Stata, or if you have access to HMD Stata files that have already been created, you can import the Stata files easily into your software environment, provided that your software can do Stata file import. There is one caveat, however: You should not directly import HMD Stata files into your software. Rather, first use hmddata use to load the HMD Stata files into Stata, save the data set in memory manually, and run the external software import routine on the file that you have created. The reason for this procedure is that HMD Stata files store the data in a transformed way that minimizes disk space usage. hmddata use undoes these data transformations.

\section{Examples}

If you wanted to generate Stata files of all HMD data, you could download http://www.mortality.org/hmd/zip/all hmd/hmd countries.zip (user name and password required), extract the source files, and run

- hmddata settings path, value(hmddatafolder)

- hmddata convert_all, source(unzippedfilesfolder)

Folder hmddatafolder will then contain roughly 80 Stata files containing all HMD data. hmddata use knows that it should access data from this folder.

In the examples below, only period life table data with a $5 \times 10$ age-by-year grid is used. Instead of downloading and converting all data, which takes time and disk space, one can download specific files (It_male.zip, It_female.zip, and It_both.zip from http://www.mortality.org/hmd/zip/by statistic/). After file extraction, the following commands create three HMD data files in myhmddatafolder:

- hmddata convert lifetable, sourcedir(unzippedfilesfolder) grid $(5 \times 10)$

The hmddata help files explain the download and conversion process in more detail.

\footnotetext{
${ }^{6}$ An installation zip file is also included with this Technical Report. Installation follows the same procedure as for the installation zip file described in the main text. However, it is highly recommended that, if you have to do a zip file install, you use the installation zip file under the address in the main text, as only this one will reflect future code updates. This is crucial e.g. for accessing data of populations that will be added to HMD in the future.
} 
We can now start working with the data. We load data for both sexes by

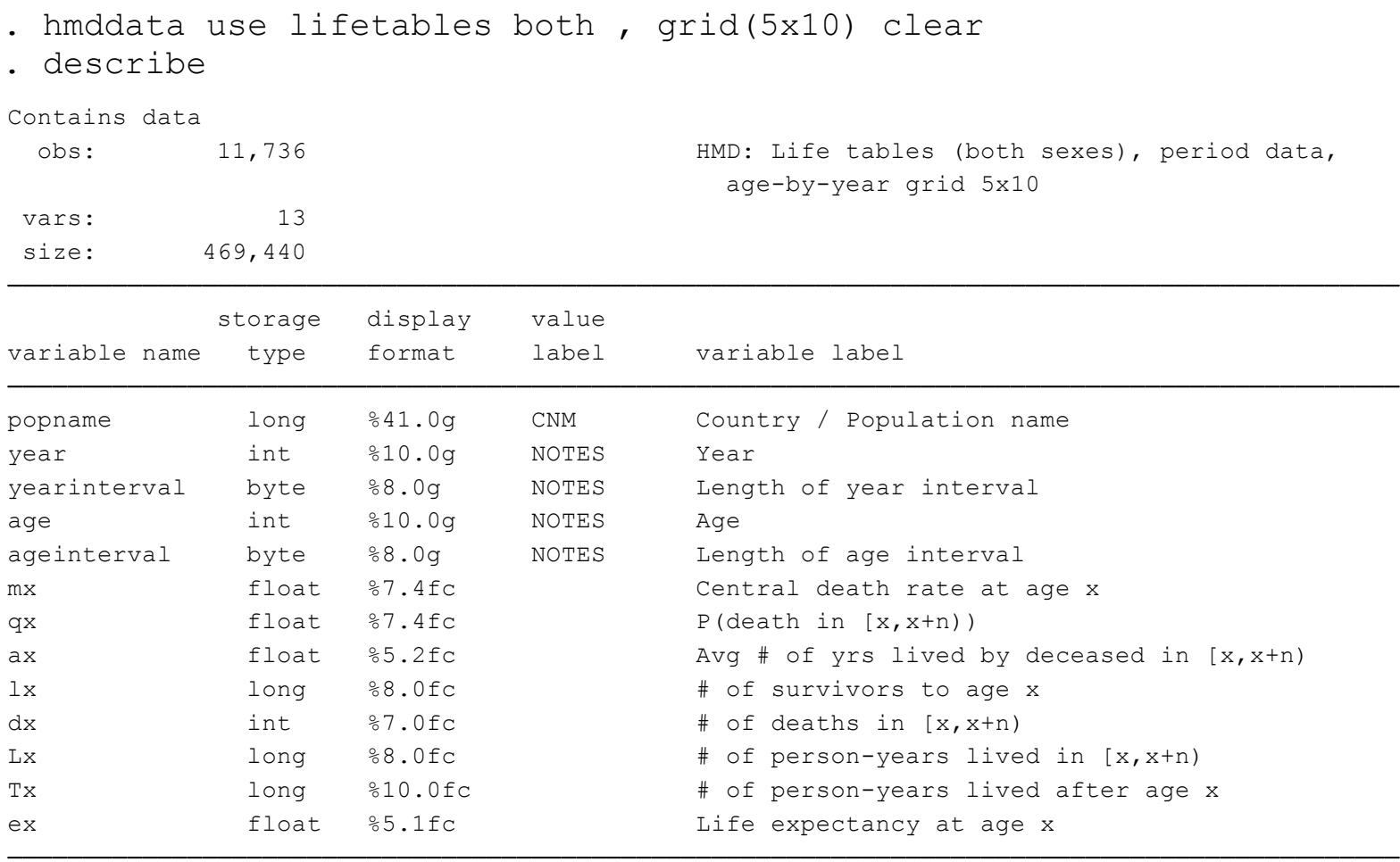

Sorted by: popname year age

The data set contains data on all HMD populations:

- tab popname

\begin{tabular}{r|rrr} 
Country / Population name & Freq. & Percent & Cum. \\
\hline U.S.A. & 168 & 1.70 & 1.70 \\
U.K.: United Kingdom Total Population & 192 & 1.95 & 3.65 \\
U.K.: Northern Ireland & 192 & 1.95 & 5.60 \\
U.K.: Scotland & 360 & 3.65 & 9.25
\end{tabular}

\section{(output omitted)}

\begin{tabular}{r|rrr} 
Latvia & 120 & 1.22 & 95.62 \\
Hungary & 144 & 1.46 & 97.08 \\
Lithuania & 120 & 1.22 & 98.30 \\
Slovenia & 48 & 0.49 & 98.78 \\
Poland & 120 & 1.22 & 100.00 \\
\hline Total & 9,864 & 100.00
\end{tabular}


The age-by-year grid is $5 \times 10$ :

- tabl yearinterval ageinterval

-> tabulation of yearinterval

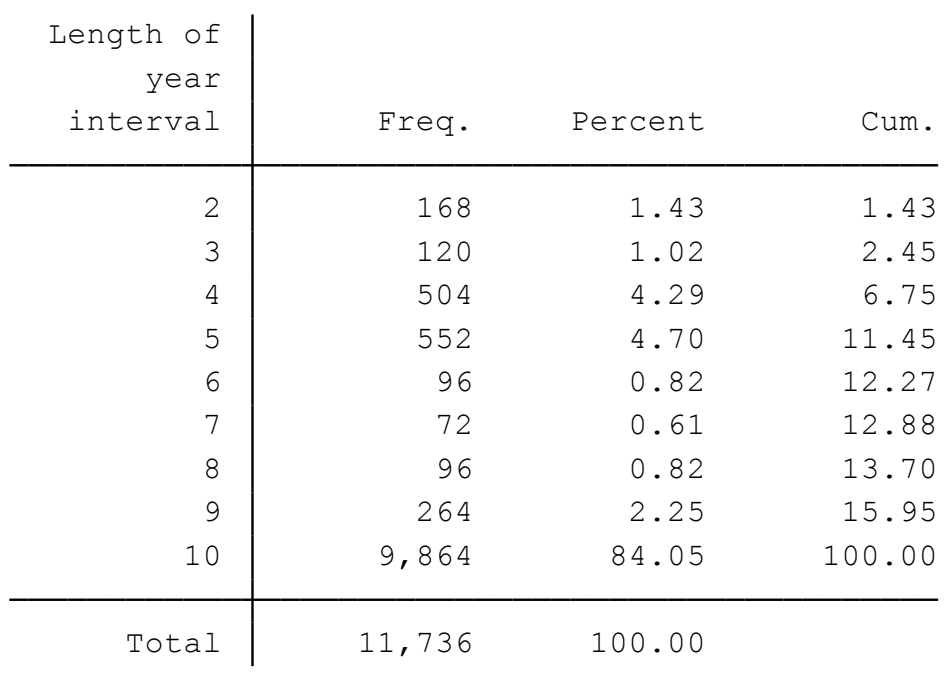

$->$ tabulation of ageinterval

\begin{tabular}{r|rrr}
$\begin{array}{r}\text { Length of } \\
\text { age }\end{array}$ & Freq. & Percent & Cum. \\
interval & 489 & 4.35 & 4.35 \\
\hline 1 & 489 & 4.35 & 8.70 \\
4 & 10,269 & 91.30 & 100.00 \\
\hline 5 & 11,247 & 100.00 &
\end{tabular}

The year interval is mostly 10, but some shorter intervals are also included in the file. The age interval follows the familiar demographic $0,1-4,5-9,10-14$, etc. age classes. Using a few preparatory statements, including one using hmddata popfilter, which filters populations, we can generate a meaningful table for a set of countries.

- hmddata popfilter esp prt ita, iso dummy (south)

- keep if yearinterval==10

- hmddata intervals agestr yearstr

- replace south $=0$ if year $<1940$ 
- table agestr popname if south \& inlist(age, 0, 1, 20, 60), contents (min $\operatorname{mx} \max m x)$

\begin{tabular}{|c|c|c|c|}
\hline $\begin{array}{l}\text { Age } \\
\text { interval }\end{array}$ & $\begin{array}{r}\text { Country } \\
\text { Italy }\end{array}$ & $\begin{array}{l}\text { / Population } \\
\text { Portugal }\end{array}$ & $\begin{array}{l}\text { name } \\
\text { Spain }\end{array}$ \\
\hline \multirow[t]{2}{*}{0} & 0.0038 & 0.0041 & 0.0037 \\
\hline & 0.1027 & 0.1335 & 0.1079 \\
\hline \multirow[t]{2}{*}{$1-4$} & 0.0002 & 0.0003 & 0.0002 \\
\hline & 0.0104 & 0.0207 & 0.0115 \\
\hline \multirow[t]{2}{*}{$20-24$} & 0.0005 & 0.0007 & 0.0005 \\
\hline & 0.0073 & 0.0045 & 0.0049 \\
\hline \multirow[t]{2}{*}{$60-64$} & 0.0078 & 0.0092 & 0.0080 \\
\hline & 0.0215 & 0.0239 & 0.0258 \\
\hline
\end{tabular}

The table contains minimum and maximum death rates for the periods covered in the data set.

As another example, we can produce a meaningful graph quickly. We look at infant death rates in Chile in this example.

- hmddata use lifetables, grid(5x10) popfilter(chile) clear

- hmddata intervals

- graph bar (asis) mx if inlist(age, 0, 1, 5) \& inlist(sex, 1, 2) , over(yearstr) over(agestr) by (sex, col(1) title(Infant Death Rates in Chile)) nofill

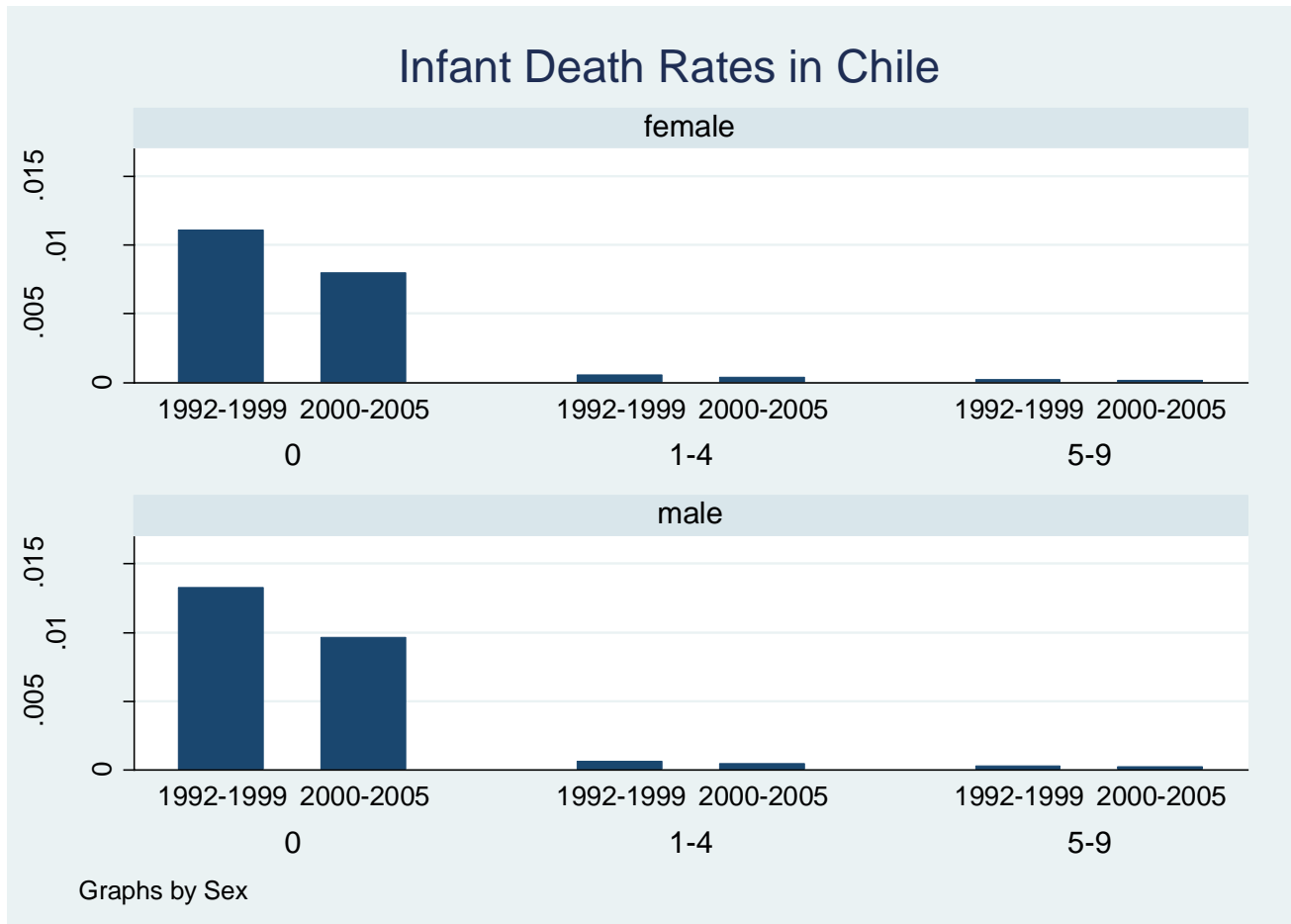

hmddata intervals generated a variable that enabled us to label the age categories nicely. 
As a second graph example, we create a scatter plot of life expectancy versus the infant mortality rate for all HMD countries. We apply different marker colors for each half-century in order to visualize the development over time.

- hmddata use lifetables bothsexes, grid(5x10) clear

- keep if $\bmod ($ year, 10) $=0$ \& yearinterval $==10$ \& age==0

A few auxiliary statements are necessary for the coloring of the marker variable:

- gen $y^{50}=\operatorname{int}(($ year-1700) / 50)

- label define y50 1 "1750-" 2 "1800-" 3 "1850-" 4 "1900-" 5 "1950-" 6 "2000-"

- label values y50 y50

- decode y50, gen (y50_str)

- bys y50 age : replace y50_str = " " if $n !=1$

- local scopts mlab(y50_str) mlabpos(2) $\bar{m} l a b g a p(* 30)$ mlabsize (huge) ylab $(30(10) 90) \quad x l a b(0(0.05) 0.4)$

The graph statement is: ${ }^{7}$
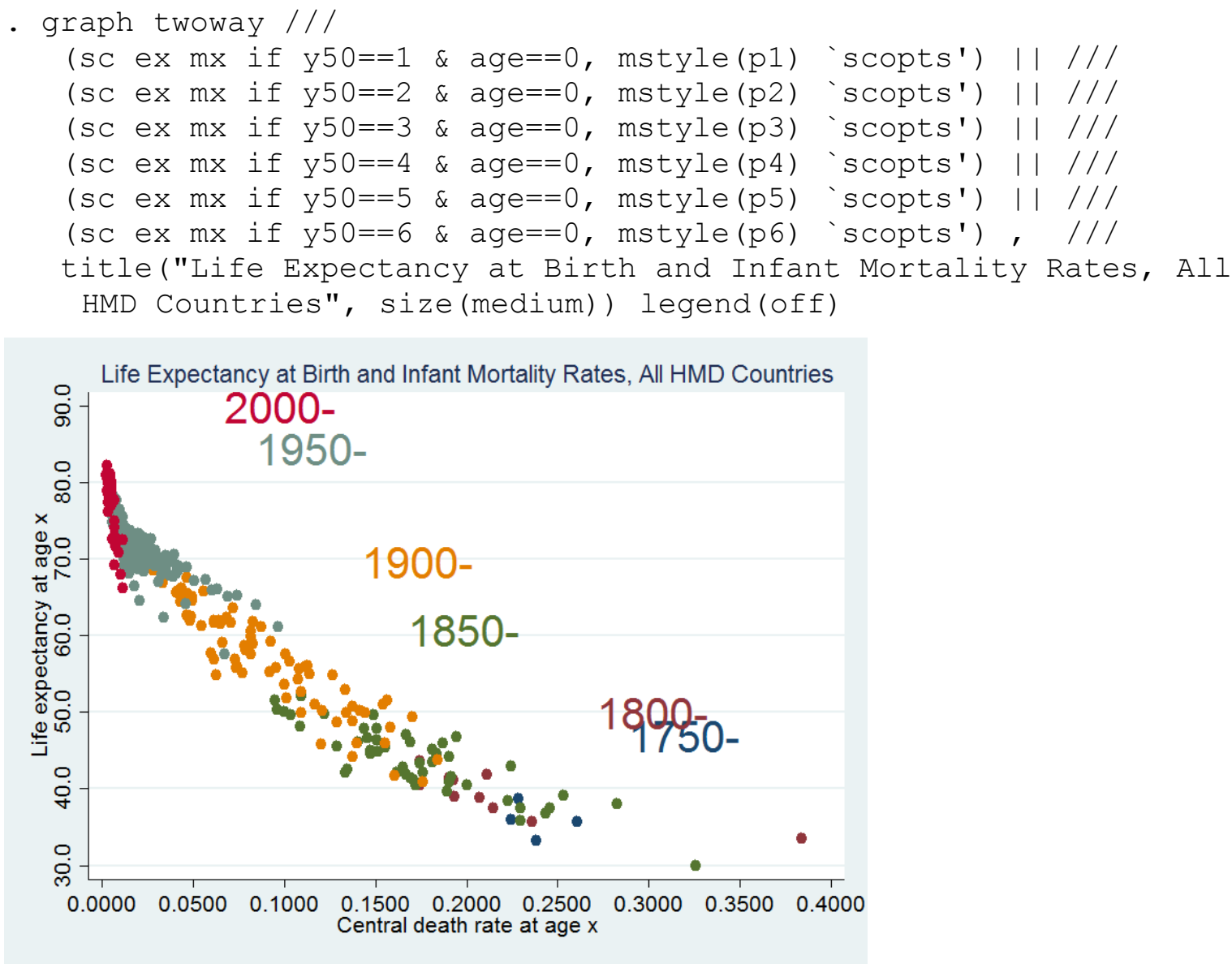

\footnotetext{
${ }^{7}$ You may have noticed that the resulting graph has an inaccurate $X$-axis title: It says "Central death rate at age $x^{\prime \prime}$, while the graph depicts death rates at age 0 . This is because the variable $\mathrm{mx}$ in the data set retrieved by hmddata use really does contain death rates at all ages ("at age $x^{\prime \prime}$ ). In the example we deleted all ages except 0 , so the variable now only contains rates at age 0 , but hmddata has no way of knowing this, so it does not delete the variable label of $m x$ which is used in the graph. This underscores that hmddata can be used to quickly generate nicely labeled tables and graphs, but that the labeling may not always be perfect. In these cases you may wish to further supply labeling information; here, using the xtitle ( ) option. Analogous comments for the $\mathrm{Y}$-axis title in the example graph apply.
} 
Up to now we have looked at graphs generated by standard Stata graph commands. We now explore the graph subcommand of hmddata, which facilitates the quick generation of graphs that are a bit more involved.

In the following, we use data from East and West Germany:

- hmddata use lifetables, grid(5x10) clear

- keep if yearinterval==10

- hmddata popfilter germanyeast germanywest, dummy(ger)

- hmddata intervals

hmddata graph provides a flexible syntax to specify dimensions and levels along which line graphs shall be generated. We look at the development of infant mortality and life expectancy over time again.

- hmddata graph line ex year if ger, at1 (age 0 1) at2 (sex female male) by (pop)

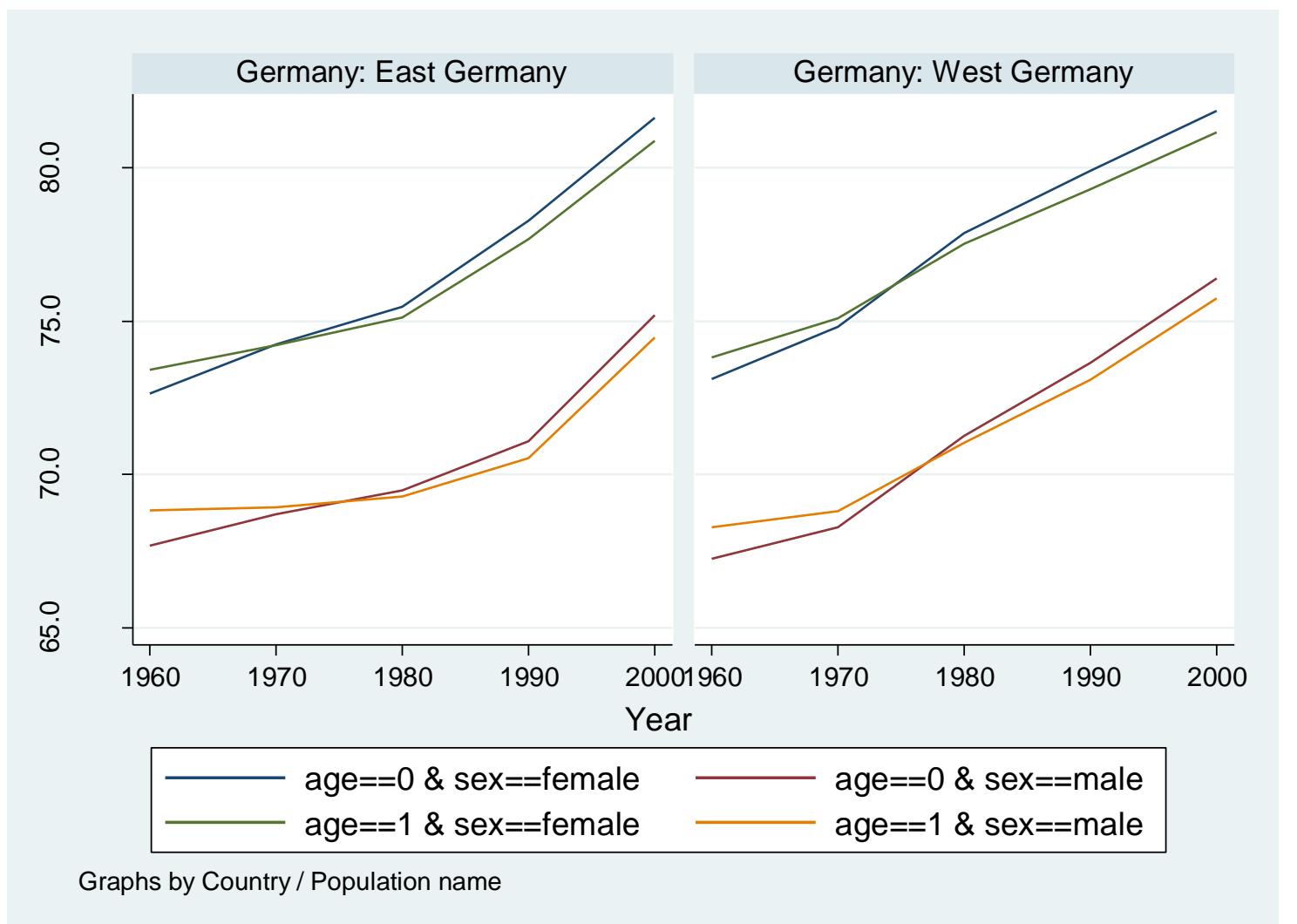


If we want to present the same information by sex instead of by region, we just have to juggle option arguments a little:

- hmddata graph line ex year if ger \& sex!=3, at1 (age 0 1) at2 (pop) by (sex, leg(col(1))) leg(size(vsmall))

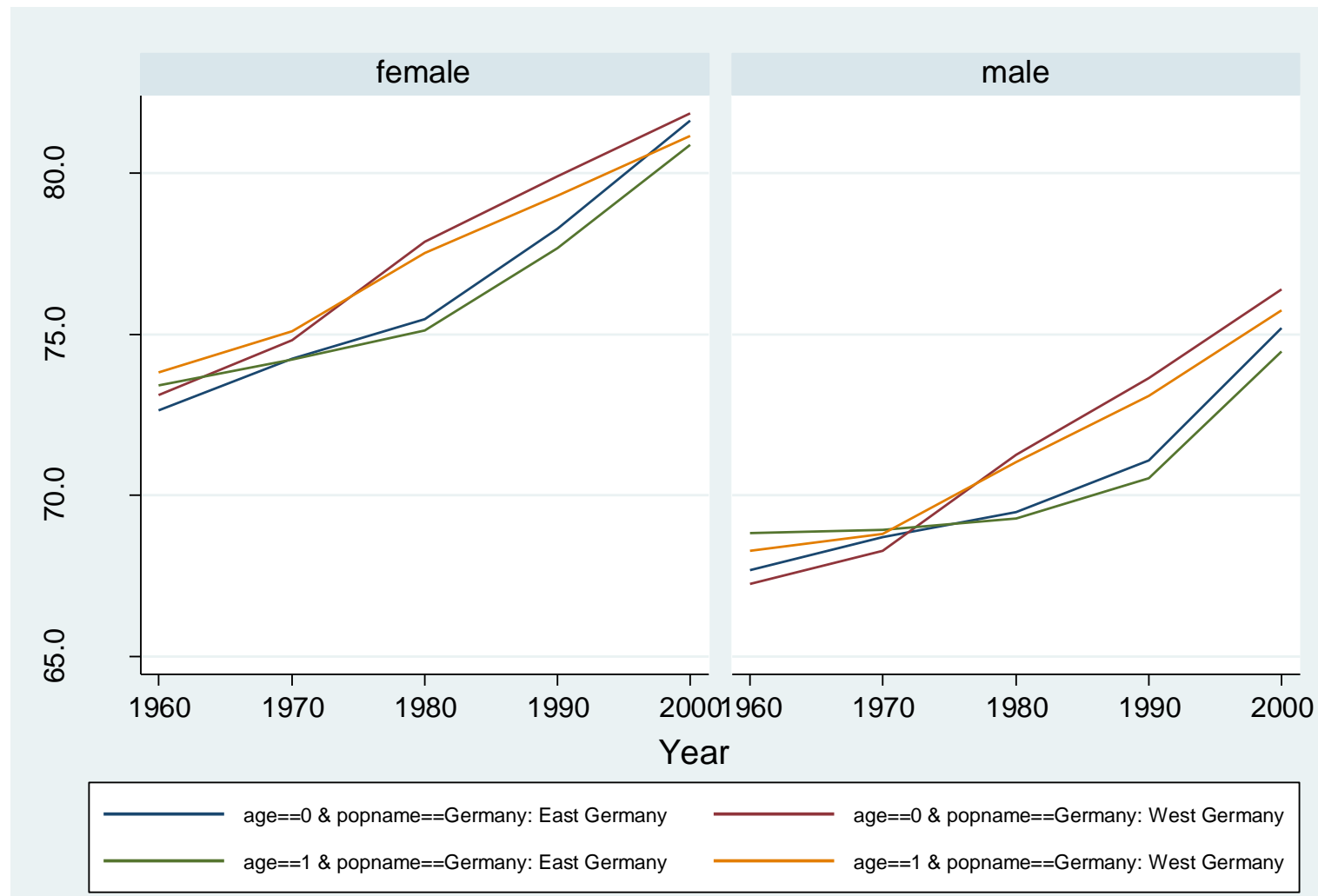

Graphs by Sex

\section{Conclusion}

This technical report introduced a Stata add-on module called "hmddata". It facilitates importing and working with data from the Human Mortality Database (HMD). Both usage of HMD data and of the hmddata tool are free of charge, and data access and installation are quick and easy. Many more details and additional topics related to hmddata, such as data updates and replicability, are discussed in the Stata help files that come with the package.

\section{Acknowledgements}

I thank Dmitri Jdanov, Tim Riffe, Vladimir Shkolnikov, and the staff of the Demographic Data Lab of the Max Planck Institute for Demographic Research for helpful comments. 


\section{References}

Human Mortality Database. University of California, Berkeley (USA) and Max Planck Institute for Demographic Research (Germany). Available at www.mortality.org and www.humanmortality.de.

Hyndman, Rob J. (2017): demography: Forecasting Mortality, Fertility, Migration and Population Data. R package version 1.20 .

Minton, Jon (2015): Merging, Exploring, and Batch Processing Data from the Human Fertility Database and Human Mortality Database. Technical Report TR-2015-001, Max Planck Institute for Demographic Research (MPIDR). URL http://www.demogr.mpg.de/papers/technicalreports/tr-2015-001.pdf.

Riffe, Tim (2015): Reading Human Fertility Database and Human Mortality Database Data into R. . Technical Report TR-2015-004, Max Planck Institute for Demographic Research (MPIDR). URL http://www.demogr.mpg.de/papers/technicalreports/tr-2015-004.pdf.

Shkolnikov, Vladimir M. and Dmitri A. Jdanov (2016): R Programs for Writing HMD Life Tables and HMD Death Rates to Pooled Data Files. Technical Report 2016-001, Max Planck Institute for Demographic Research (MPIDR). URL http://www.demogr.mpg.de/papers/technicalreports/tr2016-001.pdf. 